\title{
A novel co-locational and concurrent fNIRS/EEG measurement system: design and initial results.
}

\author{
Darren J. Leamy and Tomas E. Ward
}

\begin{abstract}
We describe here the design, set-up and first time classification results of a novel co-locational functional NearInfrared Spectroscopy/Electroencephalography (fNIRS/EEG) recording device suitable for brain computer interfacing applications using neural-hemodynamic signals. Our dual-modality system recorded both hemodynamic and electrical activity at seven sites over the motor cortex during an overt finger-tapping task. Data was collected from two subjects and classified offline using Linear Discriminant Analysis (LDA) and Leave-One-Out Cross-Validation (LOOCV). Classification of fNIRS features, EEG features and a combination of fNIRS/EEG features were performed separately. Results illustrate that classification of the combined fNIRS/EEG feature space offered average improved performance over classification of either feature space alone. The complementary nature of the physiological origin of the dual measurements offer a unique and information rich signal for a small measurement area of cortex. We feel this technology may be particularly useful in the design of BCI devices for the augmentation of neurorehabilitation therapy.
\end{abstract}

\section{INTRODUCTION}

Brain Computer Interfacing (BCI) is making steady progress towards becoming a mainstream alternative interaction technology. This progress has been driven in large part to the increasing availability of inexpensive electrophysiological apparatus and the application of ever more sophisticated signal processing methods. However, progress in the exploitation of alternative physiological measurements for such interfacing has been limited and remains an aspect of the discipline warranting more concerted investigation. In this paper we describe initial progress in the development of an alternative measurement technology.

We propose the use of neural-hemodynamic signals as an interface technology suitable for brain computer interfacing. This work is motivated by research towards the development of BCI suitable for the augmentation of stroke rehabilitation therapy. By measuring cortical activity associated with motor function for those individuals unable to engage with conventional therapy, as a consequence of little or no residual movement, it is hypothesized that increased drive to neuroplastic processes can be administered through appropriate neuro-feedback [1]. This application space presents a highly demanding environment for the required BCI technology. For example, the techniques used must be capable of generating

This work was supported by Science Foundation Ireland's Research Frontiers Program RFP0000

D. J. Leamy is with Biomedical Research Group, Department of Electronic Engineering, National University of Ireland Maynooth, Maynooth, Ireland dleamy@eeng.nuim.ie

T. E. Ward is with Biomedical Research Group, Department of Electronic Engineering, National University of Ireland Maynooth, Maynooth, Ireland tward@eeng.nuim. ie stereotypical responses which can be associated with meaningful biofeedback targets. Accuracy is also important as the signal produced must be sufficiently robust so as to minimize classification errors.

An additional concern is that the technology must be convenient, unobtrusive and provide as little discomfort as possible to the patient. It is therefore unreasonable to expect a patient to travel to a research laboratory to spend extended periods of time engaging with an uncomfortable cortical activity recording device. Consequently the technology must be capable of presentation in a small form factor, have a quick subject set-up time and should be as portable as possible.

Given the constraints already highlighted and, in particular the application area of stroke, where the pathophysiology arises from damage to the neural vasculature, we speculate that a suitable BCI technology should combine both neural and hemodynamic signatures of cortical function. Such a compound signal conveys information not only about underlying neural activity but also the associated vasculature response, changes in which may reflect structural and regenerative changes in cortex. This represents a dual modality measurement. In order to achieve this we propose the use of a combined optical and electrical measurement system, which in this case can be achieved through functional near infrared spectroscopy (fNIRS) and electroencephalography (EEG).

\section{A. Functional Near-Infrared Spectroscopy (fNIRS)}

fNIRS uses light in the near-infrared range (typically 660 $\mathrm{nm}$ to $1000 \mathrm{~nm}$ ) to measure the dynamic changes in cerebral blood oxygenation and blood flow of a localised area of the brain [2]. fNIRS uses pairs of light sources and light detectors operating at two or more discrete wavelengths. A source-detector pair is referred to as an "optode" and constitutes a single fNIRS channel. The light is emitted into the scalp of a subject from the light source, where the photons of light are either absorbed or scattered at a molecular level. A portion of the emitted photons are scattered to such a degree that they exit the scalp near to the point of entry. A light detector can be placed on the scalp to measure the intensity of the light exiting at that point. Models of propagation of near-infrared light in the human head have shown that the photons that exit at a particular point will have followed a roughly banana-shaped path, the depth of which depends on the source-detector separation [3]. 
Oxygenated hemoglobin ( $\mathrm{HbO})$ and deoxygenated hemoglobin (HbR) are two components of blood that absorb infrared and near-infrared light with known extinction coefficient spectra [4]. Therefore, the amplitude of a recorded fNIRS signal is a function of the concentration of $\mathrm{HbO}$ and $\mathrm{HbR}$ along the path of the photons. Using knowledge of the specific extinction coefficients of $\mathrm{HbO}$ and $\mathrm{HbR}$ at the wavelengths used, a measurement of the relative $\mathrm{HbO}$ and $\mathrm{HbR}$ levels can be found using analytical and/or emperical calculations [5]. fNIRS responses have been established in previous works e.g. [6] and have been utilised for BCI applications [7].

\section{B. Electroencephalography (EEG)}

Non-invasive EEG is an electrical measurement of the spatially integrated, near-synchronous dendritic activity of similarly oriented neurons near the surface of the brain. The EEG demonstrates spectral structure which can change as a consequence of neural drive associated with brain function. In particular motor tasks elicit specific well known patterns in spectra which have been used as the basis for BCI [8]. One such pattern is Event Related Synchronisation/Desynchronisation (ERS/ERD), which is a relative increase/decrease in the band power of a chosen frequency range that coincides with some event. In ERS/ERD analysis, a baseline 'reference' period of EEG data is recorded before the event and then compared to an 'activity' period of EEG data, recorded during or following the event [9]. ERD is known to occur in the $\mu$ frequency range $(8-12 \mathrm{~Hz})$ on movement onset and ERS is known to occur in the $\beta$ frequency range $(12-30 \mathrm{~Hz})$ following movement offset [10], [11].

\section{Methodology}

\section{A. Device design}

We designed a module to hold three fNIRS light sources, three fNIRS light detectors and seven EEG electrodes in the array shown in Figure 1. There are seven fNIRS channels with the corresponding EEG electrodes located directly above the centre point of each fNIRS channel. The centre point of an fNIRS channel is the interrogated area of cortex, so with our set-up, we are recording electrical and hemodynamic activity from approximately the same area of cortex. Thus, we have seven co-locational, dual-modality recording

TABLE I

EEG ELECTRODES, fNIRS SOURCES AND FNIRS DETECTORS USED FOR EACH fNIRS/EEG CHANNEL.

\begin{tabular}{|c|c|c|c|}
\hline $\begin{array}{c}\text { Channel } \\
\text { Num. }\end{array}$ & $\begin{array}{c}\text { EEG } \\
\text { Electrode }\end{array}$ & $\begin{array}{c}\text { fNIRS } \\
\text { Source }\end{array}$ & $\begin{array}{c}\text { fNIRS } \\
\text { Detector }\end{array}$ \\
\hline 1 & E1 & S1 & D1 \\
2 & E2 & S1 & D2 \\
3 & E3 & S2 & D1 \\
4 & E4 & S2 & D2 \\
5 & E5 & S3 & D2 \\
6 & E6 & S2 & D3 \\
7 & E7 & S3 & D3 \\
\hline
\end{tabular}

sites. Table I details the electrodes and optodes used for each neuro-hemodynamic channel. fNIRS data was recorded using a TechEn CW6 system (TechEn Inc., USA). Wavelengths used were $690 \mathrm{~nm}$ and $830 \mathrm{~nm}$, sampled at $25 \mathrm{~Hz}$. EEG data was recorded using a BioSemi Active-Two system (BioSemi Inc., The Netherlands). DC coupled data was recorded at $2048 \mathrm{~Hz}$.

\section{B. Experiment}

To demonstrate the classification accuracy gain of combining the feature space of fNIRS and EEG, data was collected from two healthy individuals. Both subjects gave voluntary consent. Subject A was male, 37 years old and left-handed (self-reported). Subject B was male, 26 years old and right-handed (self reported). During the experiment, the subjects were seated in a comfortable chair viewing a computer screen which presented instructions. Subjects were instructed to tap each of their fingers to their thumb on both hands. Tapping was self-paced. Individual trials lasted for 20 seconds, during which time the on-screen instruction read either 'TAP' (an 'active' trial) or 'RELAX' (a 'rest'

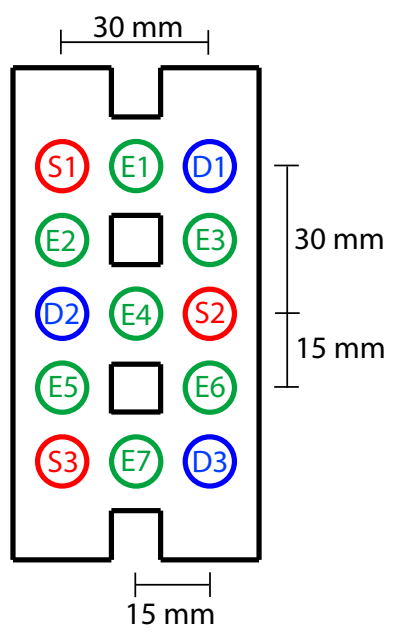

Fig. 1. Design of the dual fNIRS/EEG recording module. fNIRS sources are labelled S1 - S3. fNIRS detectors are labelled D1 - D3. EEG electrodes are labelled E1 - E7. All source-detector separations are $30 \mathrm{~mm}$. All EEG electrodes lie halfway between a source and detector.

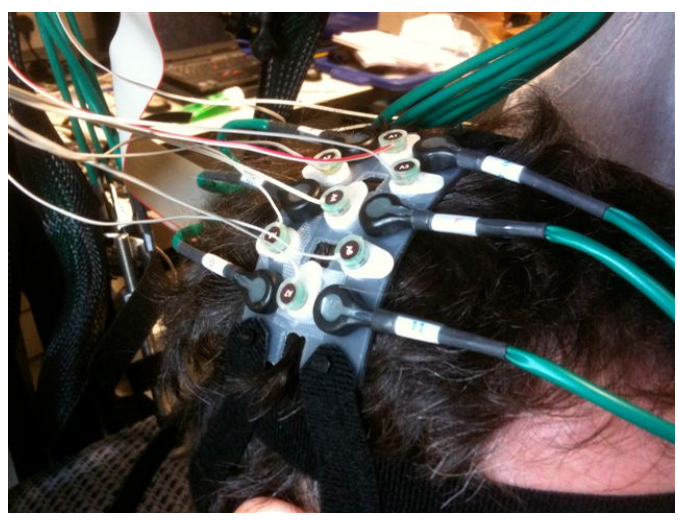

Fig. 2. Dual fNIRS/EEG module positioned over Subject A's motor cortex. 


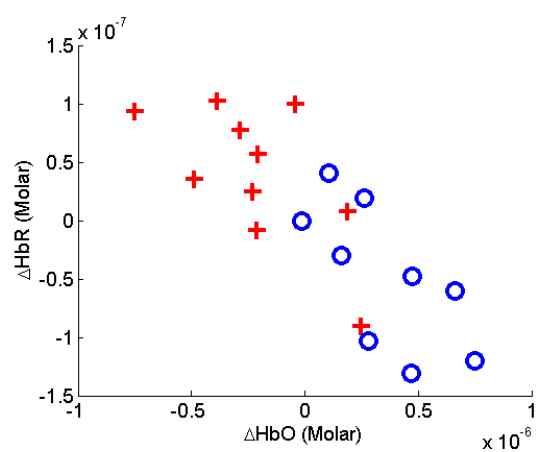

Fig. 3. 2D fNIRS feature space for Channel 2 of Subject A, Trial 1. Crosses indicate feature locations when subject is in a rest period. Circles indicate feature locations when subject is in a finger-tapping period.

trial). 20 trials were carried out per experimental run, which alternated between active and rest, lasting 400 seconds in total. Two experimental runs were recorded for each subject with a short break between runs. The central electrode of our fNIRS/EEG recording module was located at C3 for Subject A (left-handed) and C4 for Subject B (right-handed). Module position is shown in Figure 2.

\section{Signal processing}

EEG data was first analysed to identify the frequencies at which ERS and ERD occurred in the $\mu$ frequency range and $\beta$ frequency range respective to two types of event. These two events were the transition from rest to active periods and vice-versa. The frequency ranges at which ERS and ERD occurred were identified by sight by comparing average FFT plots for the reference and activity periods for both events. Raw EEG data was bandpass filtered with a 6th order Butterworth filter to the identified ERS/ERD ranges, squared to obtain a power signal and then smoothed using a lowpass 6th order Butterworth filter at $5 \mathrm{~Hz}$.

For ERS/ERD analysis, the reference window was chosen to be between 4.5 and 3.5 seconds before both types of event. For a transition from a rest trial to an active trial, the activity window was selected to be from 0 to 1 seconds after the transition. For a transition from active to rest, the activity window was selected to be from 0.5 to 1.5 seconds after the transition. These windows were chosen to capture the expected timing of pre-movement $\mu$-rhythm desynchronisation and post-movement $\beta$-rhythm synchronisation. These windowed $\mu$ and $\beta$ range power signals were used for classification of EEG activity.

For fNIRS, the $690 \mathrm{~nm}$ and $830 \mathrm{~nm}$ raw intensity measurements were first converted to changes in optical density, as per Eqn. (1a) and (1b) of [5]. Next, the Modified BeerLambert Law was applied to these signals to generate change in concentration signals for $\mathrm{HbO}$ and $\mathrm{HbR}$, as per Eqn. 3 of [5]. A differential path-length factor of 6 was used. These $\Delta \mathrm{HbO}$ and $\Delta \mathrm{HbR}$ signals were used for classification of fNIRS activity.

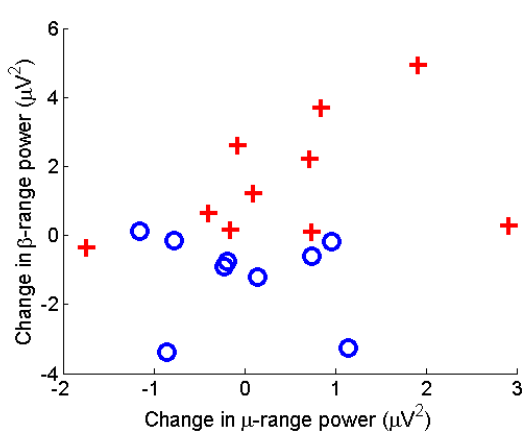

Fig. 4. 2D EEG feature space for Channel 2 of Subject A, Trial 1. Crosses indicate feature locations when subject is in a rest period. Circles indicate feature locations when subject is in a finger-tapping period.

\section{Classification}

Signal classification was performed on the fNIRS and EEG signals following the processing steps already described. The goal of classification was to decode the subject's current state based on features extracted from the fNIRS and EEG responses. We aimed to classify the activity into one of two classes: 'active' and 'rest'. We employed the Linear Discriminant Analysis (LDA) classifier and calculated classification accuracy via leave-one-out cross-validation (LOOCV). In particular, for $N$ trials, $N-1$ trials were used for training the classifier and the remaining 1 trial was used for testing. This was repeated $N$ times with each trial used for testing once. Classification accuracy was calculated as the number of correct classifications over $N$.

For EEG, the feature extracted was change in $\mu$-rhythm and $\beta$-rhythm power from the reference period to the activity period at the beginning of a trial. This resulted in a 2 dimensional EEG feature space (Figure 4). For fNIRS, the average change in amplitude of the $\Delta \mathrm{HbO}$ and $\Delta \mathrm{HbR}$ signals over a trial were used to define a 2-dimensional fNIRS feature space (Figure 3). By combining the fNIRS and EEG feature spaces, an fNIRS/EEG 4-dimensional feature space was also created for classification.

\section{RESULTS}

A table of classification results are presented in Table II. Shown is the classification accuracy of the classifier when operating on fNIRS features alone, EEG features alone and combined fNIRS/EEG features. A summary of results is presented in Table III. Subject A demonstrated an average pre-movement $\mu$-rhythm ERD in the $9-11 \mathrm{~Hz}$ range and average post-movement $\beta$-rhythm ERS in the 19$22 \mathrm{~Hz}$ range over all EEG channels. Subject B demonstrated average pre-movement $\mu$-rhythm ERD in the $9-12 \mathrm{~Hz}$ range and average post-movement $\beta$-rhythm ERS in the $19-21 \mathrm{~Hz}$ range over all EEG channels. Our results show that utilising both fNIRS and EEG features for classification yields an improvement on classification accuracy.

\section{Discussion}

Our results have shown an average increase in classification accuracy by combining fNIRS features and EEG 
TABLE II

LDA CLASSIFICATION RESULTS FOR fNIRS FEATURES ONLY, EEG FEATURES ONLY AND COMBINED fNIRS/EEG FEATURES.

\begin{tabular}{|c|c|c|c|c|c|c|c|c|c|c|c|c|}
\hline \multirow[b]{3}{*}{ Channel } & \multicolumn{6}{|c|}{ Subject A } & \multicolumn{6}{|c|}{ Subject B } \\
\hline & \multicolumn{3}{|c|}{ Trial 1} & \multicolumn{3}{|c|}{ Trial 2} & \multicolumn{3}{|c|}{ Trial 1} & \multicolumn{3}{|c|}{ Trial 2} \\
\hline & fNIRS & EEG & Comb. & fNIRS & EEG & Comb. & fNIRS & EEG & Comb. & fNIRS & EEG & Comb. \\
\hline 1 & $84 \%$ & $79 \%$ & $90 \%$ & $100 \%$ & $84 \%$ & $95 \%$ & $79 \%$ & $84 \%$ & $95 \%$ & $79 \%$ & $84 \%$ & $84 \%$ \\
\hline 2 & $79 \%$ & $79 \%$ & $84 \%$ & $95 \%$ & $79 \%$ & $95 \%$ & $47 \%$ & $79 \%$ & $63 \%$ & $53 \%$ & $84 \%$ & $84 \%$ \\
\hline 3 & $100 \%$ & $74 \%$ & $95 \%$ & $100 \%$ & $84 \%$ & $100 \%$ & $79 \%$ & $74 \%$ & $79 \%$ & $84 \%$ & $84 \%$ & $84 \%$ \\
\hline 4 & $95 \%$ & $84 \%$ & $95 \%$ & $84 \%$ & $84 \%$ & $79 \%$ & $74 \%$ & $74 \%$ & $63 \%$ & $53 \%$ & $90 \%$ & $79 \%$ \\
\hline 5 & $74 \%$ & $74 \%$ & $84 \%$ & $42 \%$ & $74 \%$ & $58 \%$ & $47 \%$ & $68 \%$ & $47 \%$ & $74 \%$ & $79 \%$ & $95 \%$ \\
\hline 6 & $100 \%$ & $90 \%$ & $100 \%$ & $95 \%$ & $74 \%$ & $90 \%$ & $58 \%$ & $74 \%$ & $68 \%$ & $79 \%$ & $84 \%$ & $79 \%$ \\
\hline 7 & $58 \%$ & $84 \%$ & $84 \%$ & $68 \%$ & $68 \%$ & $79 \%$ & $68 \%$ & $63 \%$ & $68 \%$ & $58 \%$ & $74 \%$ & $63 \%$ \\
\hline Average & $84 \%$ & $80 \%$ & $90 \%$ & $83 \%$ & $78 \%$ & $85 \%$ & $65 \%$ & $74 \%$ & $69 \%$ & $68 \%$ & $83 \%$ & $81 \%$ \\
\hline
\end{tabular}

features into a single fNIRS/EEG feature space. Results for each subject, however, were slightly different. For Subject A, the combined classifier yielded better results than either fNIRS or EEG individually, but for Subject B, results were slightly better for EEG classification than for combined classification. While this may seem discouraging, this result is not unexpected. For 2D data, LDA finds a line between classes that best describes the classification boundary. In combining two 2D feature spaces, it's possible that the hyperplane boundary does not as clearly divide the two classes. This appears to have occurred with Subject B's data. However, the average gain of using dual feature classification over fNIRS is greater than the loss when compared to EEG feature classification.

Our results suggest that classification with combined fNIRS/EEG is an improvement over fNIRS or EEG individually. Therefore, we expect a similar improvement to be seen when using dry electrodes, instead of the standard wet electrodes we have used here. Should these results hold for dry electrodes, then we could design a completely dry fNIRS/EEG recording system, which will significantly reduce set-up time and subject discomfort and may have similar classification success to an all-wet electrode EEG system.

\section{CONCLUSions}

The investigation of alternative technologies for BCI is an important endeavour if engineers are to design systems that have broader appeal and utility than current systems. Here we have successfully demonstrated the feasibility of technology yielding a neural-hemodynamic interface for the

TABLE III

SUMMARY OF CLASSIFICATION RESULTS.

\begin{tabular}{|c|l|l|l|}
\hline Subject & fNIRS & EEG & Dual \\
\hline A & $83.5 \%$ & $79 \%$ & $87.5 \%$ \\
B & $66.5 \%$ & $78.5 \%$ & $75 \%$ \\
\hline Average & $75 \%$ & $79 \%$ & $81 \%$ \\
\hline
\end{tabular}

monitoring of cortical activity associated with motor movement. We believe that this technology will facilitate further investigation into the development of easy-to-use mainstream rehabilitation technology utilising BCI principles.

\section{ACKNOWLEDGMENTS}

The authors gratefully acknowledge the contribution of Science Foundation Ireland: Research Frontiers Program 2009, Grant No. 09/RFP/ECE2376.

\section{REFERENCES}

[1] T. E. Ward and C. J. Soraghan and F. Matthews and Charles Markham, A Concept for Extending the Applicability of Constraint-Induced Movement Therapy through Motor Cortex Activity Feedback Using a Neural Prosthesis., Comput. Intell. Neurosci. vol. 2007, 2007, 51363.

[2] Y. Hoshi, Functional near-infrared spectroscopy: current status and future prospects., J. Biomed. Opt., vol. 12, 2007, pp 062106.

[3] E. Okada and M. Firbank and M. Schweiger and S. R. Arridge and M. Cope and D. T. Delpy, Theoretical and experimental investigation of near-infrared light propagation in a model of the adult head., Appl. Opt., vol. 36, 1997, pp 21-31.

[4] M. Cope, The application of near infrared spectroscopy to non invasive monitoring of cerebral oxygenation in the newbron infant., $\mathrm{PhD}$. Thesis, University College London, 1991.

[5] T. J. Huppert and S. G. Diamond and M. A. Franceschini and D. A. Boas, HomER: a review of time-series analysis methods for nearinfrared spectroscopy of the brain., Appl Opt, vol. 48, 2009, pp D280D298.

[6] T. J. Huppert and R. D. Hoge and S. G. Diamond and M. A. Franceschini and D. A. Boas, A temporal comparison of BOLD, ASL, and NIRS hemodynamic responses to motor stimuli in adult humans., NeuroImage, vol. 29, 2006, pp 368-382.

[7] F. Matthews and B. A. Pearlmutter and T. E. Ward and C. Soraghan and C. Markham, Hemodynamics for Brain-Computer Interfaces., IEEE Signal Processing Magazine, vol. 25, 2008, pp 87-94.

[8] E. Angelakis and A. Hatzis and I. G. Panourias and D. E. Sakas, Braincomputer interface: a reciprocal self-regulated neuromodulation., Acta. Neurochir. Suppl., vol. 97, 2007, pp 555-559.

[9] G. Pfurtscheller and F. H. Lopes da Silva, Event-related EEG/MEG synchronization and desynchronization: basic principles., Clinical Neurophysiology, vol. 110, 1999, pp 1842-1857.

[10] G. Pfurtscheller and A. Aranibar, Evaluation of event-related desynchronisation (ERD) preceding and following voluntary self-paced movements., Electroenceph. Clin. Neurophysiol., vol. 46, 1979, pp $138-146$

[11] G. Pfurtscheller and A. Stancák, Jr. and C. Neuper, Post-movement beta synchronization. A correlate of an idling motor area?, Electroenceph. Clin. Neurophysiol., vol. 98, 1996, pp 281-293. 